\title{
Reconfigurable Dual-Polarized Beam-steering Broadband Antenna Using a Crossed-Strips Geometry
}

\author{
Germán A. Ramírez (D), Ivan Zhou (D), Sebastian Blanch (D), Member, IEEE, Md. Asaduzzaman Towfiq (D), \\ Bedri A. Cetiner (D), Member, IEEE, Jordi Romeu (D), Fellow, IEEE, Lluis Jofre-Roca (iD Fellow, IEEE
}

\begin{abstract}
In this letter, a reconfigurable dual-polarized broadband antenna with beam-steering capabilities using a parasitic layer is proposed for 5G New Radio (NR) Frequency Range 1 (FR-1) applications. The antenna is a dual-port aperture-stacked patch structure with symmetrical orthogonal (horizontal and vertical) currents. The beam-steering is achieved by a pair of reconfigurable cross-shaped parasitic strips which bestow the antenna three main beam directions $\theta=\{\sim$ $\left.-25^{\circ}, 0^{\circ}, \sim 25^{\circ}\right\}, \phi=\left\{0^{\circ}\right\}$, with pointing and gain ( $\left.7 \mathbf{d B}\right)$ stability across a $30 \%$ impedance bandwidth $\left(S_{11} \& S_{22}<-10\right.$ dB) from $3.2-4.3 \mathbf{~ G H z}$ for both ports/polarizations. A prototype of the antenna is manufactured and measured demonstrating results in accordance with simulation expectations.
\end{abstract}

Index Terms-Multi-port antennas, Broadband antennas, Dual-polarization antennas, Reconfigurable antennas, Beam-steering antennas, 5G New Radio, FR-1 (Sub-6 GHz).

\section{INTRODUCTION}

$\mathbf{S}$ IMULTANEOUS transmission and reception of two channels of information on the same carrier frequency by using two orthogonal polarizations, is an effective way of doubling the wireless communication capacity. Supporting dual-polarization using an orthogonally dual-polarized antenna is a standard requirement for the Fifth Generation of Mobile Communications (5G) New Radio (NR) Base Station (BS) and Customer Premises Equipment (CPE) [1]. Another important feature of $5 \mathrm{G} \mathrm{NR}$ is to employ beam-steering antennas both on the user and infrastructure sides of the communication link. Beam-steering in its many variants is reckoned as an effective

Manuscript received February 14, 2020; revised April xx, 2020

This work was supported by Colombian "Departamento Administrativo de Ciencia, Tecnología e Innovación" Colciencias through convocatoria 727 of 2015 scholarship, and by the Spanish "Comision Interministerial de Ciencia y Tecnologia" (CICYT) under projects TEC2013-47360-C3-1-P/AEI/10.13039/501100011033, TEC2016-78028-C3-1-P/AEI/10.13039/501100011033, and MDM2016-0600, and Catalan Research Group 2017 SGR 219.

Germán A. Ramírez was with Universidad Nacional de Colombia, Bogotá, Colombia. He is now with Universitat Politècnica de Catalunya, Barcelona, Cat 08034 Spain. (e-mail: garamirezar@unal.edu.co).

Md. Asaduzzaman Towfiq is with i5 Technologies, Inc. Utah State University, Innovation campus, Logan, UT, 84341 (email: asaduzzaman.towfiq@i5technologies.com)

Bedri Cetiner is with Utah State University, Logan, USA. (e-mail: bedri.cetiner@usu.edu).

Ivan Zhou, Sebastián Blanch, Jordi Romeu and Lluìs. Jofre, are with Universitat Politècnica de Catalunya, Barcelona, Cat 08034 Spain. (e-mail: sebastian.blanch@upc.edu, jordi.romeu-robert@upc.edu, luis.jofre@upc.edu). technique for increasing capacity and energy efficiency, as well as reducing interference [2] in mobile communications.

Furthermore, it is foreseen that a large portion of the initial deployments of the $5 \mathrm{G}$ NR will be concentrated on the Frequency Range 1 (FR-1) (sub-6 GHz portion of the spectrum), and special interest is raised on the n77 band, comprised between $3.3-4.2 \mathrm{GHz}$, where moderately large bandwidths up to $100 \mathrm{MHz}$ can be allocated.

The dual-polarized antenna presented in this work combines the above mentioned three properties of 5G NR, namely dual-polarization, beam-steering, and broad bandwidth, into a single reconfigurable antenna architecture. Accomplishing all these properties by a low-complex and -cost antenna architecture is the main novelty of this work. The reconfigurability in polarization and beam-steering is achieved by employing the reconfigurable Parasitic Layer (PL) approach [3]. This approach separates the driven antenna from the reconfigurable layer, thus has the advantage of playing minimum impact on the antenna parameters non intended to be modified. This concept has been successfully used to add beam-steering capabilities to microstrip patch antennas, and proven to be an appealing alternative to traditional beamformers, keeping a low cost and complexity [4].

Reconfigurable PL structures of large and medium complexity [5], [6] have been used to add polarization shifting to single-port antennas, where a large number of switching elements is used. While most approaches have focused on beam-steering rather than on bandwidth, the later is often not a major concern given the rich reconfigurability potential of PL approach. For example, the work in [6] achieves concurrently reconfiguring frequency, polarization, and pattern properties of a base patch antenna albeit by using excessively large number of switching elements. Cost and reliability concerns associated with using a large number of switches have led to recent modifications of the PL concept aimed at reducing the number of switches and thus the complexity and cost of manufacturing and maintenance. Two recent examples of simplified PLs are [7], [8] presenting antenna designs conceived upon the Yagi-Uda parasitic array concept.

The antenna presented in this work is novel in that it provides beam-steering capabilities to a dual-polarized broadband antenna in three main lobe directions $\left(\phi=0^{\circ}, \theta \in\right.$ $\left\{0^{\circ}, \sim \pm 25^{\circ}\right\}$ ) based on a low-complexity PL concept. This new PL structure is based on a pair of reconfigurable 


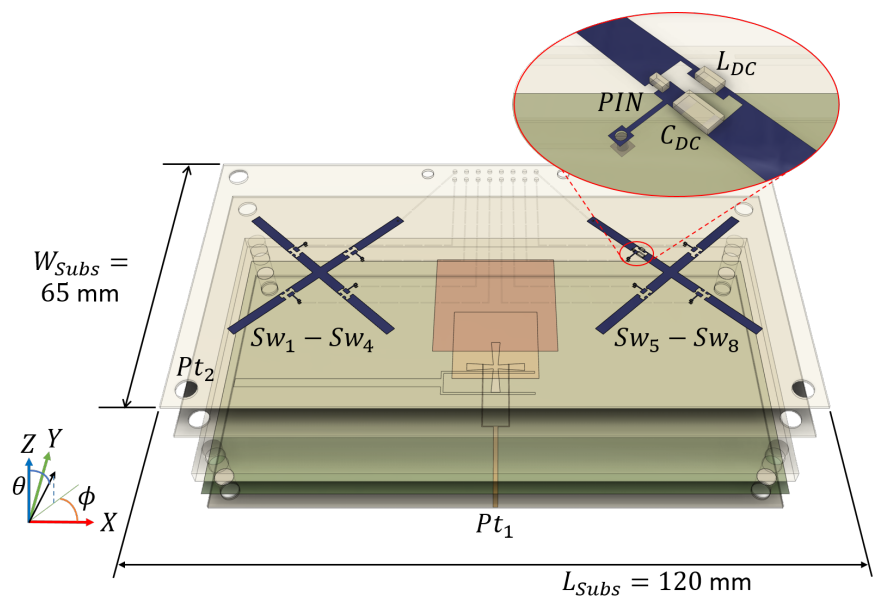

Fig. 1: Antenna schematic - Exploded view.

cross-shaped parasitic strips designed in a way that can simultaneously interact with currents in two polarizations without significantly altering antenna matching. Additionally, given the orientation of the crossed-strips relative to the base antenna currents, a bow-tie effect [9] is achieved which is essential for accomplishing a large beam-steering bandwidth. Therefore, this new PL features also pointing and gain stability across its impedance bandwidth as well as high port (cross-polarization) isolation.

The presented antenna has a simple switching scheme, where only two control lines are needed to excite six different modes of operation at an individual antenna level.

\section{ANTENNA STRUCTURE AND WORKING PRINCIPLE}

An exploded view of the antenna structure is illustrated in Fig. 1. where substrates, support posts and biasing lines are drawn translucent for visualization clarity. The antenna is composed by six copper layers, etched on five substrates all of them Rogers RO4003C $\left(\epsilon_{r}=3.55, \tan \delta=0.0027\right)$. The substrate thicknesses from bottom to top are $h_{s_{i}}=$ $\{0.508,0.508,3.0,0.508,0.508\} \mathrm{mm},(i=1, . ., 5)$. The first three substrates are stacked with ideally no separation in between, whereas the fourth and fifth are separated from the previous one by air gaps with heights of $\{8.0$, and 6.0$\} \mathrm{mm}$, respectively. These air gaps are provided by $4 \times \varnothing 6 \mathrm{~mm}$ plastic (nylon) supports. The overall height of the antenna stack-up is $h=19.03 \mathrm{~mm}$, which is about one-fifth of the wavelength at the lowest operating frequency of $3.2 \mathrm{GHz}$.

To accomplish the first two operational requirements of dual-port polarization diversity and broadband operation, an Aperture-Stacked Patch Antenna (ASP) [10]-[12] is designed as base radiator. This base radiator has similar match and radiation properties at both ports/polarizations by evenly exciting squared patches through an asymmetric cross-shaped slot. For the sake of simplicity, the antenna is fed by two orthogonal microstrip lines matched with dual-offset end-lines.

Once the base antenna has been designed with satisfactory performances in terms of bandwidth and port isolation, the next step is to introduce the beam-steering capabilities for both polarizations. Beam-steering needs to be accomplished
TABLE I: Antenna operation modes.

\begin{tabular}{c|c|cccccccc} 
Mode & $(\theta, \phi)_{\max }$ & $S_{1}$ & $S_{2}$ & $S_{3}$ & $S_{4}$ & $S_{5}$ & $S_{6}$ & $S_{7}$ & $S_{8}$ \\
\hline$M_{1}$ & $(0,0)$ & 0 & 0 & 0 & 0 & 0 & 0 & 0 & 0 \\
$M_{2}$ & $(25,0)$ & 1 & 1 & 1 & 1 & 0 & 0 & 0 & 0 \\
$M_{3}$ & $(-25,0)$ & 0 & 0 & 0 & 0 & 1 & 1 & 1 & 1
\end{tabular}

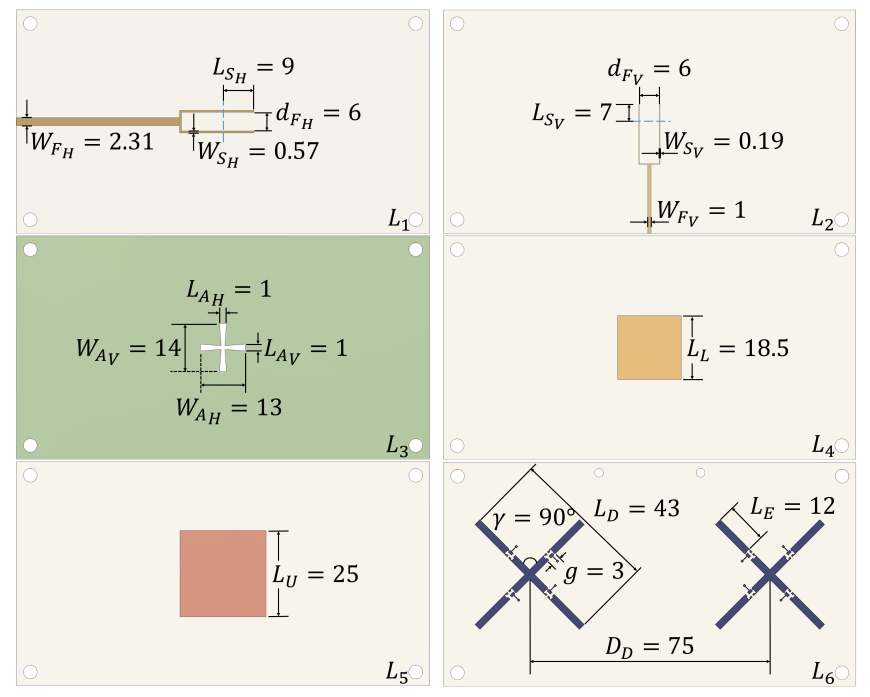

Fig. 2: Antenna layers and parameter values (in $\mathrm{mm}$ ).

for each port of orthogonal polarization while maintaining the desired broad bandwidth and ports isolation. This is achieved by the addition of a reconfigurable PL on top, which consists of a pair of cross-shaped strips with properly located switches. The base antenna together with the PL generates three main beam directions $\left(\phi=0^{\circ}, \theta \in\left\{0^{\circ}, \sim \pm 25^{\circ}\right\}\right)$, by the corresponding switching combinations summarized in Table. [1

Figure. 2 illustrates a layer-by-layer view of the antenna including its relevant parameters and respective values (all in $\mathrm{mm}$ ). These values have been obtained by performing parametric Full-Wave Electromagnetic (FWEM) simulations using the CST time domain solver, which has taken into consideration broadband performance metrics related to port match, angular deviation, and gain.

The functioning of the parasitic layer can be explained in terms of parasitic Yagi-Uda like arrays, which consists of driven and loaded reflector/director elements [7], [13] (switches loads are properly accounted for both "ON" and "OFF" states). In this structure, the dual-port ASP acts as the driven element, whereas the cross-shaped parasitic elements may act either as a reflector or a director depending on the length, angle and separation of them. When all the switches $\left(\left\{S w_{1}-S w_{4}\right\}\right.$ or $\left.\left\{S w_{5}-S w_{8}\right\}\right)$ are in the "ON" state, significant currents in both horizontal and vertical directions are induced making them act as reflectors for both polarizations. When all switches are in the "OFF" state, the size of the crossed-shaped parasitic element is scaled down, which becomes mostly transparent for both currents of orthogonal polarizations. Therefore, when all switches are "OFF" the beam will point in broadside direction.

It is noteworthy to mention that the presented steering principle is independent of the basic antenna, and although the ASP antenna used in this work has an inherently broad 


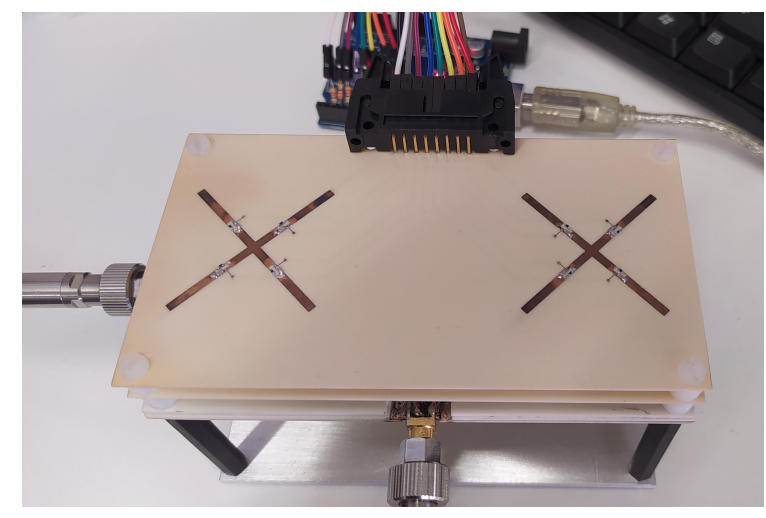

Fig. 3: Manufactured antenna prototype ready for radiation pattern measurements. The metal plate at the bottom, separated $50 \mathrm{~mm}$ away from the bottom plate of the antenna, is used to affix the antenna to the anechoic chamber support.

beamwidth $\left(80^{\circ}\right)$, the benefits of the underlying reconfigurable technique will be more remarkable when combined with narrow beamwidth antennas where the gain margin at the defection angles will be higher compared to that of the basic antenna.

Three additional advantages of this design are, first that thanks to the crossed-strips configuration both polarizations can be steered in the same direction without modifying the states of the switches, providing an agility factor in Multiple-Input Multiple-Output (MIMO) and diversity applications. Second is the low complexity achieved, as even though there are 8 switches, only 2 control lines are required greatly simplifying the biasing network. Finally, as shown in [8], [14], [15] the non-linear effects of the switching diodes are minimized when they are included in the parasitic elements rather than in the active element, as for a given transmitted power the currents in the parasitic elements are smaller than those of the active element. Also noteworthy is that steering in the $\phi=90^{\circ}$, and $\phi= \pm 45^{\circ}$ planes could also be achieved by replicating the crossed-strips with a $\phi=90^{\circ}$ rotation, similar to the concept presented by the authors in [16], thus achieving a total of 9 modes of operation for each feeding port/polarization combination.

\section{RESUlts}

A prototype of the antenna, illustrated in Fig. 3, was manufactured using mainstream Printed Circuit Board (PCB) technology, and measured at the Universitat Politècnica de Catalunya (UPC) facilities. Switches are implemented with the AlGaAs Flip Chip MA4AGFCP910 PIN diodes, using 15 $\mathrm{nH}$ inductors (LQW15AN15NG00D) and $1.2 \mathrm{pF}$ capacitances (CBR06C129B5GAC) to form the bias Tee. Similarly, DC ground reference is delivered to each part of the reconfiguring strip using identical $15 \mathrm{nH}$ inductors. Switches are controlled by using an Arduino Uno board. Also, aiming to minimize air gaps between the stacked substrates a thin film of $3 \mathrm{M}$ spray mount was used to join the first three layers.

As a reference, S-parameters of the basic antenna without the PL (BA), and the complete reconfigurable antenna

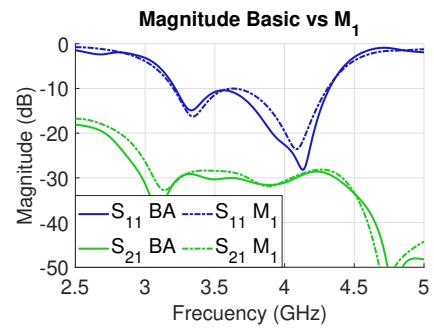

(a)

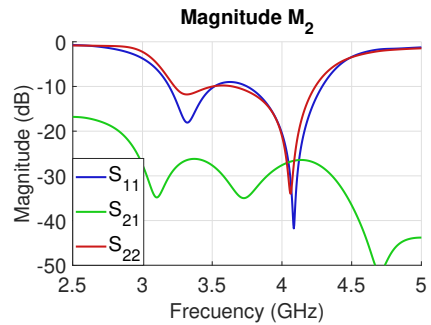

(b)
Fig. 4: Antenna port parameters (Measured). (a) Basic (BA) and complete antenna in $M_{1}$. (b) Complete antenna $M_{2}$.

operating in Mode $1\left(M_{1}\right.$ : All switches are in "OFF" state) have been measured and these results are compared with the simulations. These reference results are shown in Fig. 4a where good input ports' matching, $S_{11} \& S_{22}<-10 \mathrm{~dB}$, and high port isolation of $S_{21} \sim-30 \mathrm{~dB}$ are achieved from 3.2 to $4.3 \mathrm{GHz}$. The measured input matching and port isolation results for Mode $2\left(M_{2}:\left\{S w_{1}-S w_{4}\right\}\right.$ are in "ON" state) are shown in Fig. $4 \mathrm{~b}$ Comparing the results in Fig. $4 \mathrm{a}$ and Fig. $4 b$ reveals that the parasitic layer do not have an adverse impact on the port isolation and input matching over the whole bandwidth. A high port isolation $S_{21}<-25 \mathrm{~dB}$ demonstrates that the cross-shaped parasitic strip do not have a strong influence on cross-polarization from the antenna ports point of view. Similar results are obtained for Mode 2 and Mode 3 $\left(M_{3}:\left\{S w_{5}-S w_{8}\right\}\right.$ are in "ON" state).

To investigate the broadband beam-steering capabilities of the proposed reconfigurable antenna design, radiation patterns for modes $M_{1-3}$ at five frequency samples $f_{i}=$ $\{3.3,3.5,3.7,3.9,4.1\} \mathrm{GHz}$, are measured in the $\phi=0$ plane (with orthogonal polarizations coplanar to the currents excited when feeding each of the input ports). The measured results are compared with those obtained from simulation, and the radiation patterns at central frequency are presented in Fig. 5. The measured and simulated results agree well, where good pattern shape, beam pointing stability, and symmetrical behavior of modes $M_{2}$ and $M_{3}$ are observed within the frequency band of interest. In all the cases, co-pol component is verified to be at least more than $15 \mathrm{~dB}$ above the cross-pol component. An average gain improvement of $2.8 \mathrm{~dB}$ is observed at the angles where maximum steering occurs when compared with the base antenna which is in accordance with the $3.2 \mathrm{~dB}$ predicted by the simulation.

Another metric of interest related to the radiation pattern is the gain in the direction of the main lobe. For port one, mean gains of $6.9 \mathrm{~dB}$ for $M_{1}$ and $8.2 \mathrm{~dB}$ for mode $M_{2}$ and $M_{3}$ are observed. For port two, $8.0 \mathrm{~dB}$ and $8.2 \mathrm{~dB}$ for $M_{1}$ and $M_{2-3}$ are observed, respectively. The results for Mode 3 at port 2 are shown in Fig. 6 this port is chosen as representative as it is coplanar with the plane in which scanning occurs. It is also worth mentioning that mean loss efficiencies $\left(\eta_{l}\right)$ of $87 \%$ and $89 \%$ are observed in simulation when feeding port one and two, respectively, which are consistent with the measured ones $(80 \%$ and $81 \%$ ). Furthermore, for the steering modes, i.e., Mode 2 and Mode 3, a very good 

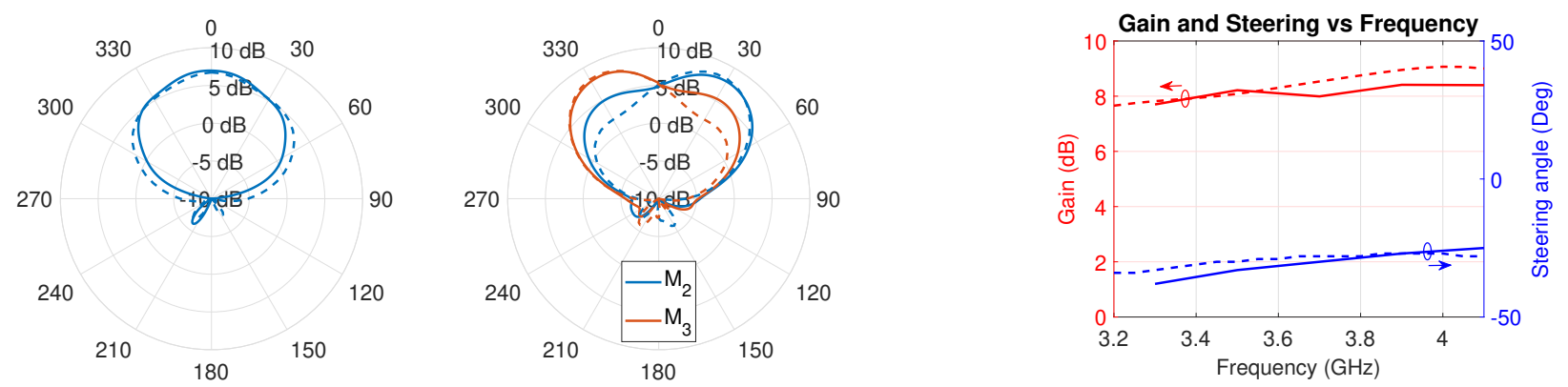

Fig. 5: Gain patterns at central frequency $f_{c}=3.7 \mathrm{GHz}$. Fig. 6: (a) Antenna Gain (Red). (b) Antenna Steering (Blue). (a) $\quad M_{1}$ (Left). (b) $M_{2}, M_{3}$ (Right). (- Measured, vs frequency (Port 2, $M_{3}$ ).( - Measured, --- Simulation.) --- Simulation.)

TABLE II: Comparison to other RA. (\#Sw: Number of switches, \#Ctrl: Number of control lines, \#Pt: Number of ports, Is: Isolation (dB), BW: Bandwidth (GHz), \%: Percent bandwidth, 2-Pol: Dual-polarized (y/n), Av. G: Average Gain (dB), Steer: Beam steering angle (deg), $\eta$ : Efficiency (\%)), Vol: Occupied volume (wavelengths at central frequency)

\begin{tabular}{c|ccccccccc} 
Ref. & \# Sw & \# Ctrl & \# Pt (Is) & BW (\%) & 2-Pol & Av G & Steer & $\eta$ & Vol. \\
\hline This work & 8 & 2 & $2(25)$ & $3.3-4.3(24)$ & Yes & 7 & $\sim 25$ & 80 & $1.5 \times 0.81 \times 0.24$ \\
{$[\overline{[7]}$} & 6 & 6 & 1 & $4.9-5.1(4)$ & No & 9 & 30 & 81 & $1.12 \times 1 \times 0.18$ \\
{$[\overline{8}]$} & 17 & 17 & 1 & $3.1-3.9(23)$ & No & 9 & 30 & NA & $1.05 \times 1.05 \times 0.25$ \\
{$[\overline{[6}$} & 60 & 60 & 1 & $2.4-3.0(22)$ & Yes & 4 & 30 & 50 & $2.16 \times 1.08 \times 0.05$ \\
{$[\overline{17}]$} & 2 & 2 & 1 & $5.1-5.9(15)$ & No & 6.5 & 30 & NA & $1.23 \times 1.1 \times 0.28$ \\
{$[\overline{18}]$} & 8 & 2 & $2(20)$ & $3.3-3.5(6)$ & Yes & 6.5 & $\sim 15$ & 70 & $1.13 \times 1.13 \times 0.08$ \\
{$[\overline{19}]$} & 4 & 2 & $2(10)$ & $2.1-2.6(20)$ & Yes & NR & 25 & NA & $1.25 \times 1.1 \times 0.23$
\end{tabular}

pointing stability $\left(\theta \sim \pm 25^{\circ}\right)$ across the frequency range of interest is achieved as shown in Fig. 6 A moderate pointing variation can be appreciated in the measurement, which is within $5^{\circ}$ of deviation from the mean value. The observed beam-squinting is in accordance with the electrical inter-cross frequency change resulting in a theoretical beam-squint within $3^{\circ}$ of deviation, which is confirmed in the very stable pointing predicted by the simulation.

A slightly larger steering angle is observed for port two which is mostly attributable to the larger portion of the ground plane associated to the radiation in the horizontal direction.

An alternative to reduce this beam-squinting effect is to search the space of individual switches states considering frequency sub-band divisions and port independence. In this way, optimum combinations ensuring even more stable pointing can be determined for a given direction. However the resulting number of switching states $\cup\left\{p_{i}, f_{n}\right\}$ (where $\left|p_{i}\right|=2$ is the number of ports, and $\left|f_{n}\right|$ is the number of sub-bands), can be as large as $\left|p_{i}\right| \times\left|f_{n}\right|$ configurations in the case that no redundancy be found, hence losing the simplicity of the control scheme herein proposed and the port switching agility.

Finally, a comparison of the introduced reconfigurable parasitic layer to other designs reported in literature is summarized in Table. [I] As can be inferred, the combination of critical features of 5G NR presented in this work, namely i) dual-polarization with two independent ports having an isolation larger than $25 \mathrm{~dB}$, ii) average beam-steering of $25^{\circ}$, and iii) broad bandwidth of $24 \%$, are achieved by the use of a simple reconfigurable architecture requiring only 2 biasing lines and 8 switches. Those features are scarcely addressed in literature, with most related works focusing only on one or two of the aspects of the proposed design, for example [18], [19] present two-ports designs, however, the former has a reduced bandwidth and beam-steering, while the later has a low port isolation and does not provide measured results. Measured average Gain and efficiency of $7 \mathrm{~dB}$ and $80 \%$, respectively, also add to the novelty and interest of this work.

\section{CONCLUSiOns}

A novel dual-polarization, broadband antenna, with beam-steering capabilities based on a reconfigurable parasitic layer approach has been designed, fabricated, and measured. The proposed technique is based on the use of a pair of cross-shaped parasitic strips interacting with currents in orthogonal directions therefore satisfactorily steering the antenna beam for its two ports of orthogonal polarizations over a broad bandwidth from 3.2 to $4.3 \mathrm{GHz}$. The measured results of the prototype antenna agreed well with simulated results showing a good gain and pointing stability over the whole bandwidth. One remarkable feature of the design approach is that all the benefits and features can be realized with a simple switching scheme that allows controlling the antenna with only two command lines. This is achieved because both ports can radiate in the same direction by using the same switches state, which also provides an agility factor to the beamforming capabilities. The presented reconfigurable antenna, which combines broadband, polarization diversity, and beam-steering capabilities into a single antenna element, can be used to form low-cost reconfigurable antenna arrays with low-power consumption, which efficiently enables achieving higher communication capacity for 5G NR FR-1 applications. 


\section{REFERENCES}

[1] 3GPP TS 38.104 V15.5.0, "Base station (bs) radio transmission and reception (release 15)," 3rd Generation Partnership Project, Technical Specification, March 2019.

[2] I. Uchendu and J. R. Kelly, "Survey of beam steering techniques available for millimeter wave applications," Progress In Electromagnetics Research B, vol. 68, pp. 35-54, May 2016.

[3] D. Rodrigo, Y. Damgaci, M. Unlu, B. A. Cetiner, J. Romeu, and L. Jofre, "Antenna reconfigurability based on a novel parasitic pixel layer," in Proceedings of the 5th European Conference on Antennas and Propagation (EUCAP), April 2011, pp. 3497-3500.

[4] Z. Li, E. Ahmed, A. M. Eltawil, and B. A. Cetiner, "A beam-steering reconfigurable antenna for wlan applications," IEEE Transactions on Antennas and Propagation, vol. 63, no. 1, pp. 24-32, Jan 2015.

[5] X. Yuan, Z. Li, D. Rodrigo, H. S. Mopidevi, O. Kaynar, L. Jofre, and B. A. Cetiner, "A parasitic layer-based reconfigurable antenna design by multi-objective optimization," IEEE Transactions on Antennas and Propagation, vol. 60, no. 6, pp. 2690-2701, June 2012.

[6] D. Rodrigo, B. A. Cetiner, and L. Jofre, "Frequency, radiation pattern and polarization reconfigurable antenna using a parasitic pixel layer," IEEE Transactions on Antennas and Propagation, vol. 62, no. 6, pp. 3422-3427, June 2014.

[7] M. A. Towfiq, I. Bahceci, S. Blanch, J. Romeu, L. Jofre, and B. A. Cetiner, "A reconfigurable antenna with beam steering and beamwidth variability for wireless communications," IEEE Transactions on Antennas and Propagation, vol. 66, no. 10, pp. 5052-5063, Oct 2018.

[8] M. A. Towfiq, A. Khalat, S. Blanch, J. Romeu, L. Jofre, and B. A. Cetiner, "Error vector magnitude, intermodulation, and radiation characteristics of a bandwidth- and pattern-reconfigurable antenna," IEEE Antennas and Wireless Propagation Letters, vol. 18, no. 10, pp. 1956-1960, Oct 2019.

[9] G. H. Brown and O. M. Woodward, "Experimentally determined radiation characteristics of conical and triangular antennas," $R C A$ Review, vol. 13, no. 4, pp. 425-452, 1952.

[10] S. D. Targonski, R. B. Waterhouse, and D. M. Pozar, "Design of wide-band aperture-stacked patch microstrip antennas," IEEE Transactions on Antennas and Propagation, vol. 46, no. 9, pp. 1245-1251, Sep. 1998.
[11] Shi-Chang Gao, Le-Wei Li, Mook-Seng Leong, and Tat-Soon Yeo, "Dual-polarized slot-coupled planar antenna with wide bandwidth," IEEE Transactions on Antennas and Propagation, vol. 51, no. 3, pp. 441-448, March 2003.

[12] S. Gao, L. W. Li, M. S. Leong, and T. S. Yeo, "A broad-band dual-polarized microstrip patch antenna with aperture coupling," IEEE Transactions on Antennas and Propagation, vol. 51, no. 4, pp. 898-900, April 2003.

[13] R. Harrington, "Reactively controlled directive arrays," IEEE Transactions on Antennas and Propagation, vol. 26, no. 3, pp. 390-395, 1978.

[14] G. A. Ramírez, J. L. Araque, C. Ballesteros, S. Blanch, J. Romeu, B. Cetiner, and L. Jofre, "Study of interconnecting switch currents in reconfigurable parasitic layer antennas," in 2019 IEEE International Symposium on Antennas and Propagation and USNC-URSI Radio Science Meeting, 2019, pp. 313-314.

[15] G. A. Ramírez Arroyave, A. Barlabé, L. Pradell, J. L. Araque Quijano, B. A. Cetiner, and L. Jofre-Roca, "Design of minimum nonlinear distortion reconfigurable antennas for next-generation communication systems," Sensors, vol. 21, no. 7, 2021. [Online]. Available: https://www.mdpi.com/1424-8220/21/7/2557

[16] I. Zhou, G. A. Ramírez, L. Montero, S. Blanch, J. Romeu, and L. Jofre, "Three-dimensional beamsteering low-complexity reconfigurable multilevel antenna," IEEE Antennas and Wireless Propagation Letters, vol. 19, no. 6, pp. 1017-1021, 2020.

[17] Z. Li, I. Bahceci, and B. A. Cetiner, "Broadband beam-steering reconfigurable antenna," Microwave and Optical Technology Letters, vol. 59, no. 1, pp. 63-65, 2017. [Online]. Available: https: //onlinelibrary.wiley.com/doi/abs/10.1002/mop.30223

[18] W. Deng, X. Yang, C. Shen, J. Zhao, and B. Wang, "A dual-polarized pattern reconfigurable yagi patch antenna for microbase stations," IEEE Transactions on Antennas and Propagation, vol. 65, no. 10, pp. 5095-5102, Oct 2017.

[19] Guo-Lin Li, X. Yang, and Cong-Song Shen, "A dual-polarized patch antenna with pattern reconfigurable characteristics," in 2014 IEEE International Conference on Communiction Problem-solving, Dec 2014, pp. 194-196. 